\title{
Effects of surface oxidation on the linear optical properties of $\mathrm{Cu}$ nanoparticles
}

\author{
Ovidio Peña-Rodríguez ${ }^{1,2, *}$ and Umapada Pal $^{3}$ \\ ${ }^{1}$ Centro de Microanálisis de Materiales (CMAM), Universidad Autónoma de Madrid (UAM), \\ Cantoblanco, E-28049 Madrid, Spain \\ ${ }^{2}$ Instituto de Óptica, Consejo Superior de Investigaciones Científicas (CSIC), C/ Serrano 121, E-28006 Madrid, Spain \\ ${ }^{3}$ Instituto de Física, Benemérita Universidad Autónoma de Puebla, Apartado Postal J-48, Puebla, Puebla 72570, Mexico \\ *Corresponding author: ovidio@bytesfall.com
}

Received June 17, 2011; revised September 12, 2011; accepted September 22, 2011; posted September 26, 2011 (Doc. ID 149449); published October 24, 2011

\begin{abstract}
Applications of copper nanoparticles (NPs) are restricted due to their proneness to oxidation by ambient oxygen if not properly protected. Here we discuss the optical properties and application potential of copper NPs covered by a thin oxide layer. Considering $\mathrm{Cu} @ \mathrm{Cu}_{2} \mathrm{O}$ core-shell type structures with different core size and shell thicknesses, linear optical properties of surface-oxidized copper NPs have been studied theoretically. Contrary to common perception, it has been demonstrated that surface-oxidized copper NPs have certain advantages for plasmonic applications. While the position of the surface plasmon resonance (SPR) can be fine-tuned by varying the thickness of the oxide layer, their plasmonic response can be enhanced (SPR intensity gain up to $30 \%$ ) by adjusting the thickness of the oxide layer. (C) 2011 Optical Society of America
\end{abstract} OCIS codes: $\quad 050.6624,160.3900,160.4236,160.4760,240.6680$.

\section{INTRODUCTION}

Metal nanoparticles (NPs) have been extensively studied in recent years due to their wide range of potential applications [1-6]. For instance, they can be used for cancer treatments $[\underline{7}, \underline{8}]$, catalysis $[\underline{9}, \underline{10}]$, photo-heat conversion [11] , surfaceenhanced Raman scattering [12], second harmonic generation [13] and biological or chemical sensing [14]. Moreover, the significant increase in the local electric field produced by the surface plasmon resonance (SPR) [15] is useful for several linear [16] and nonlinear [17-19] optical processes. Copper is an important metal because of its high electrical and thermal conductivities [20,21]. However, for applications in plasmonics, it has lagged behind gold and silver, which are usually the preferred materials for this task, due to their intense SPR and the chemical stability of the former [22-25]. Regarding the latter factor, it is well known that the surface of copper NPs gets oxidized when they are exposed to air, resulting in a core-shell structure [26,27].

On the other hand, cuprous oxide $\left(\mathrm{Cu}_{2} \mathrm{O}\right)$, the most abundant copper oxide, is a $p$-type semiconductor with a direct bandgap of $2.0 \mathrm{eV}$ [28], frequently used for solar energy conversion, electronics, magnetic storage, and gas sensing [21]. Moreover, while the surface oxidation of copper is often seen as something negative [29-31], the oxide layer stabilizes the $\mathrm{NP}$, making it more durable, and, as will be demonstrated in this work, there are at least two additional advantages of this effect: (i) the position of the SPR can be tuned by varying the thickness of the oxide layer and (ii) for certain values of the thickness of the oxide layer, the SPR intensity increases (rather than decreases, as one would expect) with respect to the Cu-only configuration, making these structures more attractive for plasmonic applications.

In this paper, we have used classical Mie calculations [32-34] to study $\mathrm{Cu} @ \mathrm{Cu}_{2} \mathrm{O}$ core-shell NPs. The bulk values of $\mathrm{Cu}$ dielectric functions reported by Johnson and Christy [35] were used to calculate their optical responses after applying the usual size correction [36,37]. The bulk optical properties of cuprous oxide [38] were used without modification. It has been observed that core-shell configurations with a ratio between the thickness of the oxide shell and the total radius of the NP around 0.2 are particularly advantageous, as the SPR intensity can be increased considerably. These metalsemiconductor structures could be used as excellent replacements for $\mathrm{Au}$ and $\mathrm{Ag}$ structures in some plasmonic applications.

\section{PROCEDURE}

In the present article, we study the linear optical response of metal-semiconductor $\left(\mathrm{Cu} @ \mathrm{Cu}_{2} \mathrm{O}\right)$ structures with geometries as shown in Fig. 1. The analytical solution for light scattering by a multilayered sphere can be found by expressing the electromagnetic field inside each layer of the sphere as a linear combination of the inward- and outward-traveling waves. Each layer is characterized by a size parameter $x_{l}=$ $2 \pi n_{m} r_{l} / \lambda=k r_{l}$ and a relative refractive index $m_{l}=n_{l} / n_{m}$, $l=1,2, \ldots, L$, where $\lambda$ is the wavelength of the incident wave in vacuum, $r_{l}$ is the outer radius of the $l$ th layer, $k$ is the propagation constant, and $n_{m}$ and $n_{l}$ are the refractive index of the medium outside the particle and its $l$ th component, respectively. Then, for an $L$-layered sphere, there are $2 L$ unknown field coefficients. The scattering coefficients can be calculated by matching the tangential components of the electromagnetic fields at each interface and solving the obtained linear system of $2 L$ independent equations [34,39].

The extinction efficiencies for the different configurations were calculated by means of ScattNLay [34], a computer implementation of the algorithm developed by Yang [39] for the calculation of the scattering of electromagnetic radiation by a 


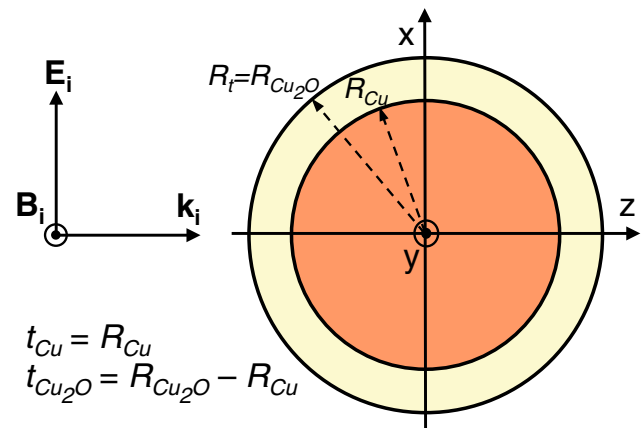

Fig. 1. (Color online) Schematic representation of the studied $\mathrm{Cu} @ \mathrm{Cu}_{2} \mathrm{O}$ structures, showing the impinging electromagnetic waves. $\mathbf{E}_{\mathbf{i}}$ and $\mathbf{B}_{\mathbf{i}}$ are the electric and magnetic fields, respectively, and $\mathbf{k}_{\mathbf{i}}$ is the wave vector.

multilayered sphere. The calculations were performed for both small (total radius, $R_{t}$, of $10 \mathrm{~nm}$ ) and big $(50 \mathrm{~nm})$ particles. In order to study the largest possible number of cases, we kept $R_{t}$ constant and varied $t_{\mathrm{Cu}_{2} \mathrm{O}} / R_{t}$ between 0.0 and 1.0. It is noteworthy that these configurations are not only of theoretical interest, but are attainable experimentally. For example, $\mathrm{Cu} @ \mathrm{Cu}_{2} \mathrm{O}$ core-shell structures with core radii of $4-5 \mathrm{~nm}$ and shell thicknesses of $2-5 \mathrm{~nm}$ are stable, and, in fact, they have been synthesized already [10].

Finally, the electric field in a particle with a spherical core of radius $R_{\mathrm{Cu}}$ and a spherical shell of radius $R_{\mathrm{Cu}_{2} \mathrm{O}}$ in a suspending medium was modeled using the quasi-static approximation $[36,40]$. When the incident field is polarized in the direction of the $X$ axis, the local electric fields in each region of the core-shell structures can be derived from Laplace's equation with the appropriate boundary conditions [40]:

$$
\vec{E}_{1}=\frac{9 \varepsilon_{\mathrm{Cu}_{2} \mathrm{O}} \varepsilon_{m}}{\varepsilon_{\mathrm{Cu}_{2} \mathrm{O}} \varepsilon_{a}-2 \varepsilon_{m} \varepsilon_{b}} E_{0}(\cos \theta \hat{r}+\sin \theta \hat{\theta})
$$

$$
\begin{aligned}
\vec{E}_{2}= & \frac{3 \varepsilon_{m}}{\varepsilon_{\mathrm{Cu}_{2} \mathrm{O}} \varepsilon_{a}-2 \varepsilon_{m} \varepsilon_{b}} \\
& \times E_{0}\left\{\begin{array}{l}
{\left[\left(\varepsilon_{\mathrm{Cu}}+2 \varepsilon_{\mathrm{Cu}_{2} \mathrm{O}}\right)+2\left(\varepsilon_{\mathrm{Cu}}-\varepsilon_{\mathrm{Cu}_{2} \mathrm{O}}\right)\left(R_{\mathrm{Cu}} / r\right)^{3}\right] \cos \theta \hat{r}+} \\
+\left[\left(\varepsilon_{\mathrm{Cu}}+2 \varepsilon_{\mathrm{Cu}_{2} \mathrm{O}}\right)-\left(\varepsilon_{\mathrm{Cu}}-\varepsilon_{\mathrm{Cu}_{2} \mathrm{O}}\right)\left(R_{\mathrm{Cu}} / r\right)^{3}\right] \sin \theta \hat{\theta}
\end{array}\right\},
\end{aligned}
$$

$$
\begin{aligned}
\vec{E}_{3}= & \left\{2 \frac{\varepsilon_{\mathrm{Cu}_{2} \mathrm{O}} \varepsilon_{a}-\varepsilon_{m} \varepsilon_{b}}{\varepsilon_{\mathrm{Cu}_{2} \mathrm{O}} \varepsilon_{a}-2 \varepsilon_{m} \varepsilon_{b}}\left(\frac{R_{\mathrm{Cu}_{2} \mathrm{O}}}{r}\right)^{3}+1\right\} E_{0} \cos \theta \hat{r} \\
& +\left\{\frac{\varepsilon_{\mathrm{Cu}_{2} \mathrm{O}} \varepsilon_{a}-\varepsilon_{m} \varepsilon_{b}}{\varepsilon_{\mathrm{Cu}_{2} \mathrm{O}} \varepsilon_{a}-2 \varepsilon_{m} \varepsilon_{b}}\left(\frac{R_{\mathrm{Cu}_{2} \mathrm{O}}}{r}\right)^{3}-1\right\} E_{0} \sin \theta \hat{\theta}
\end{aligned}
$$

where

$$
\begin{gathered}
\varepsilon_{a}=\varepsilon_{\mathrm{Cu}}(3-2 P)+2 \varepsilon_{\mathrm{Cu}_{2} \mathrm{O}} P, \\
\varepsilon_{a}=\varepsilon_{\mathrm{Cu}} P+\varepsilon_{\mathrm{Cu}_{2} \mathrm{O}}(3-P), \\
P=1-\left(R_{\mathrm{Cu}} / R_{\mathrm{Cu}_{2} \mathrm{O}}\right)^{3},
\end{gathered}
$$

where $P$ is the ratio of the shell volume to the total particle volume and $\varepsilon_{\mathrm{Cu}}, \varepsilon_{\mathrm{Cu}_{2} \mathrm{O}}$ and $\varepsilon_{m}$ are the dielectric functions of copper, cuprous oxide, and the embedding medium, respectively.

\section{RESULTS AND DISCUSSION}

In Fig. 2 we can see that by increasing the thickness of the oxide layer two different effects occur. On the one hand, the SPR redshifts, and on the other hand, the plasmon becomes more intense (with a maximum gain of $25 \%$ ) until the ratio between the thickness of the oxide shell and the total radius of the NP reaches 0.2 . From this point onward, the intensity of the SPR decreases. A similar behavior (maximum gain of 30\%) is also exhibited by the larger structures (Fig. 3), demonstrating that the effects of the oxide shell are relatively independent of the size of the particle (i.e., the phase retardation effects present in the bigger particles only play a relatively minor paper in the observed SPR variations). The described redshift can be rationalized by considering the SPR of the whole system as a homogeneous electron gas oscillating over a fixed positive background, with the induced surface charges providing the restoring force. When the semiconductor layer is added to the metallic NP, it polarizes in response to the resulting field, effectively reducing the strength of the surface charge, leading to a decreased restoring force, and, consequently, lowering the SPR energy; this effect is stronger for larger $\mathrm{Cu}_{2} \mathrm{O}$ layers. It should be mentioned that there are other ways to shift the $\mathrm{Cu}$ SPR outside the region of interband transitions [37, $\underline{41}, \underline{42}]$; however, the $\mathrm{Cu}_{2} \mathrm{O}$ layer has the added advantage of simultaneously protecting the particle.

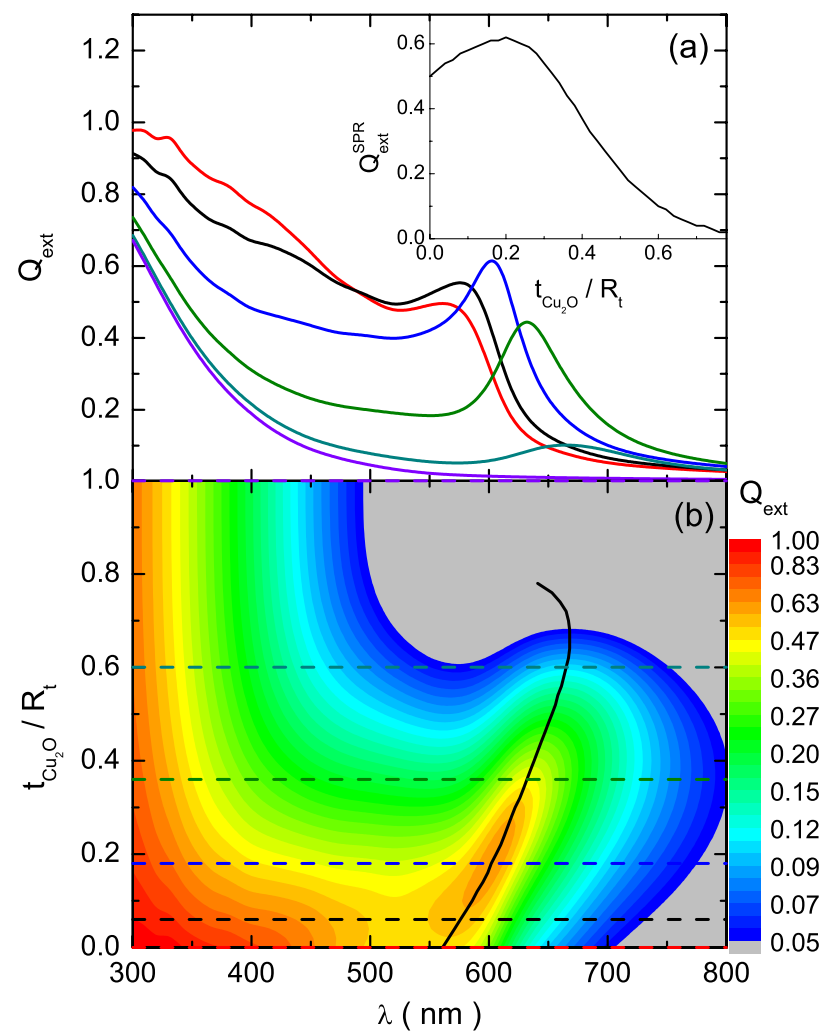

Fig. 2. (Color online) Simulated extinction efficiency for a $\mathrm{Cu} @ \mathrm{Cu}_{2} \mathrm{O}$ $\mathrm{NP}$, having $R_{t}=10.0 \mathrm{~nm}$, while $t_{\mathrm{Cu}_{2} \mathrm{O}} / R_{t}$ varies between 0.0 and 1.0 . For clarity, $Q_{\text {ext }}$ is presented for some selected values of $t_{\mathrm{Cu}_{2} \mathrm{O}} / R_{t}$ (a) and for the whole interval (b). The spectra shown in (a) correspond to the points marked with dashed lines in (b). Inset, intensity of the SPR peak versus $t_{\mathrm{Cu}_{2} \mathrm{O}} / R_{t}$. 


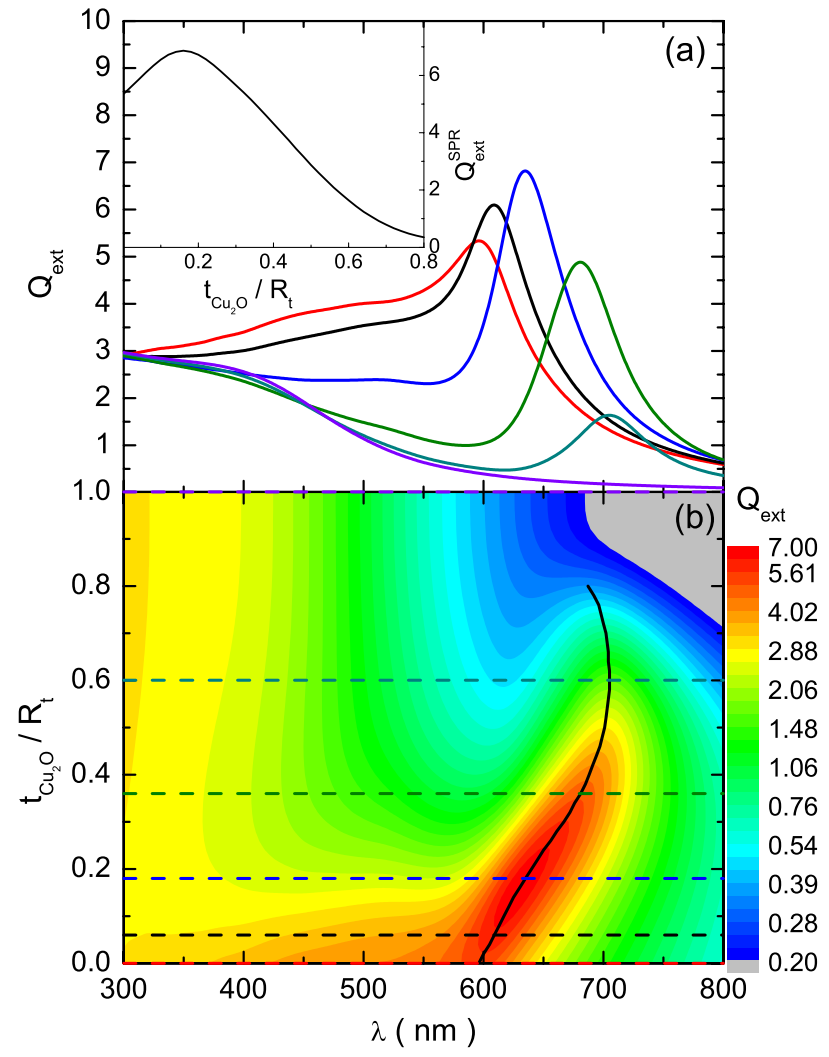

Fig. 3. (Color online) Simulated extinction efficiency for a Cu@ $\mathrm{Cu}_{2} \mathrm{O}$ structure with $R_{t}=50.0 \mathrm{~nm}$, while $t_{\mathrm{Cu}_{2} \mathrm{O}} / R_{t}$ varies between 0.0 and 1.0. For clarity, $Q_{\text {ext }}$ is presented for some selected values of $t_{\mathrm{Cu}_{2} \mathrm{O}} / R_{t}$ (a) and for the whole interval (b). The spectra shown in (a) correspond to the points marked with dashed lines in (b). Inset, intensity of the SPR peak versus $t_{\mathrm{Cu}_{2} \mathrm{O}} / R_{t}$.

Now, concerning the initial increase and subsequent decrease in the intensity of the plasmon, the effect can be understood by considering two different phenomena: the screening produced by the semiconductor layer, which tends to reduce the intensity of the SPR, and the shift of the SPR peak outside the region of interband transitions (wavelengths below $580 \mathrm{~nm}$ [37]), which makes the plasmon more intense. In other words, the SPR peak in a Cu-only NP is located inside the region of interband transitions of copper and consequently suffers a strong damping due to its interaction with the absorption background, resulting in a decrease of intensity. When the position of the SPR is shifted outside this region, the damping disappears and the peak gets more intense. Considering these two effects together, the initial gain in SPR intensity is due to the reduction of the damping but afterward, on increasing $t_{\mathrm{Cu}_{2} \mathrm{O}}$, the screening produced by the oxide layer becomes more important and the overall SPR intensity falls. It is worth noting that, in a recent independent study, Rice et al. [27] have found the same trends that we obtained theoretically. However, they assumed that the redshift and gain in intensity of the SPR were simply produced by an increase of the solvent's dielectric function due to the presence of $\pi$ bonds, overlooking the effect of the oxide layer.

Another important factor that we must consider is the intensity of the local electric field because, as we mentioned earlier, it is essential for many applications of metal NPs. In order to visualize how the presence of the $\mathrm{Cu}_{2} \mathrm{O}$ shell affects the local electric field, the local field distributions at
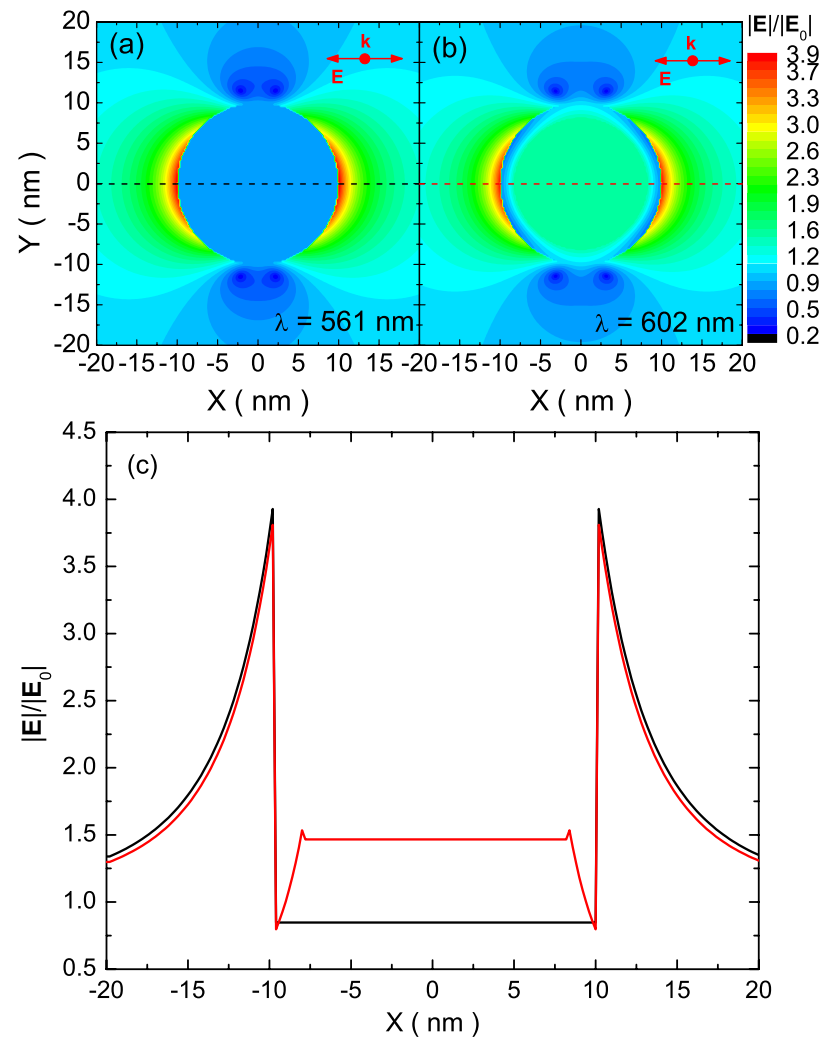

Fig. 4. (Color online) Local field contour plot in the section plane for a Cu NP with a radius of $10.0 \mathrm{~nm}$ (a) and $\mathrm{Cu} @ \mathrm{Cu}_{2} \mathrm{O} \mathrm{NP}$ with $R_{\mathrm{Cu}}=$ $8.0 \mathrm{~nm}$ and $R_{t}=10.0 \mathrm{~nm}$ (b). (c) The field profiles marked with dashed lines in the contour plots are also shown for clarity.

the resonance wavelength for the small $\left(R_{t}=10 \mathrm{~nm}\right)$ metal particle and the optimal core-shell structure (i.e., the one with the more intense SPR) are presented in Fig. 4 . As can be seen, the internal electric fields of the configurations are quite different, being about 1.5 times more intense for the NP with the oxide shell. However, the electric field is hardly affected outside the particles; its intensity at the surroundings of both structures is roughly four times more intense than that of the incident field. These results indicate that the core-shell structures enhance the local electric field as much as their metal-only counterparts; i.e., the former can be used for the same applications as the latter.

We have also studied the effects of the surrounding medium on the extinction spectra of the $\mathrm{Cu}$ and $\mathrm{Cu} @ \mathrm{Cu}_{2} \mathrm{O}$ structures (Fig. 5). The calculations were performed for a $\mathrm{Cu}$ NP with a radius of $50 \mathrm{~nm}$ and for the core-shell configuration with the highest SPR $\left(R_{\mathrm{Cu}}=42 \mathrm{~nm}\right.$ and $\left.t_{\mathrm{Cu}_{2} \mathrm{O}}=8 \mathrm{~nm}\right)$. In both cases, the SPR redshifts upon increasing the refractive index of the suspending medium. However, the observed sensitivity $\left(\Delta \lambda_{\mathrm{SPR}} / \Delta n_{m}\right)$, expressed in nanometers per refractive index units (RIUs), is not linear, and two different regimes of variation can be distinguished. For $n_{m}<1.5$ (i.e., for most liquids) the solid $\mathrm{Cu} \mathrm{NP}$ exhibits a lower sensitivity $(86.3 \mathrm{~nm} / \mathrm{RIU})$ than the $\mathrm{Cu} @ \mathrm{Cu}_{2} \mathrm{O}$ structure $(100.3 \mathrm{~nm} / \mathrm{RIU})$, while for greater values of $n_{m}$ the tendency is reversed, obtaining values of $231.1 \mathrm{~nm} / \mathrm{RIU}$ and $185.1 \mathrm{~nm} / \mathrm{RIU}$ for $\mathrm{Cu}$ and $\mathrm{Cu} @ \mathrm{Cu}_{2} \mathrm{O}$, respectively. The obtained results indicate that for most practical cases, it would be more convenient to use the $\mathrm{Cu} @ \mathrm{Cu}_{2} \mathrm{O}$ structures for optical sensing applications instead of the solid $\mathrm{Cu}$ spheres. However, sensitivity is not the only factor 


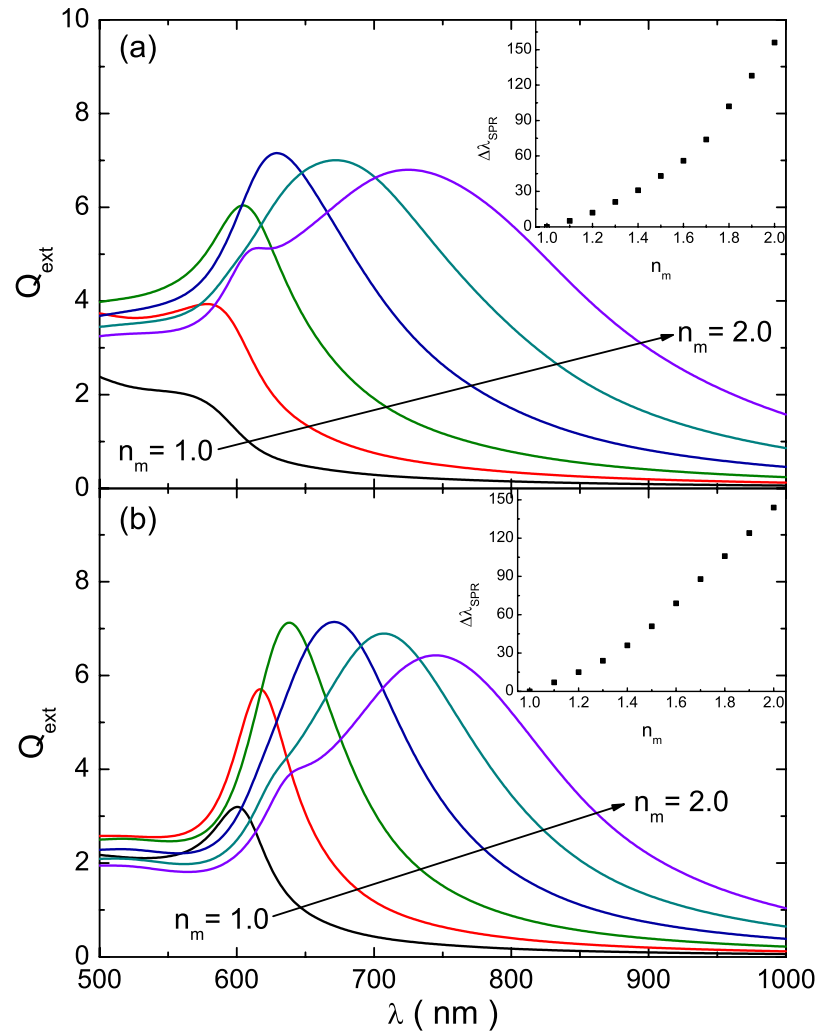

Fig. 5. (Color online) Simulated optical extinction spectra for a $\mathrm{Cu}$ $\mathrm{NP}$ with a radius of $50.0 \mathrm{~nm}$ (a) and $\mathrm{Cu} @ \mathrm{Cu}_{2} \mathrm{O} \mathrm{NP}$ with $R_{\mathrm{Cu}}=42.0 \mathrm{~nm}$ and $R_{t}=50.0 \mathrm{~nm}(\mathrm{~b})$, embedded in different media with increasing refractive indices (1.0-2.0). Insets, plots of SPR peak position $\lambda_{\mathrm{SPR}}$ against the refractive index of the embedding medium.

to consider when evaluating the merits of a certain structure for sensing applications, because the peak width and intensity also affect the ability to detect variations in the SPR position. Then, a more realistic comparison requires the use of a figure of merit (FOM) $[43,44]$ that takes into consideration those three factors:

$$
\mathrm{FOM}=\frac{\left\langle Q_{\mathrm{ext}}^{\mathrm{SPR}}\right\rangle}{\langle\mathrm{FWHM}\rangle} \frac{\Delta \lambda_{\mathrm{SPR}}}{\Delta n_{m}},
$$

where $\left\langle Q_{\mathrm{ext}}^{\mathrm{SPR}}\right\rangle$ and $\langle\mathrm{FWHM}\rangle$ are the average values of the extinction efficiency at the SPR wavelength and the full-width at the half-maximum of the SPR peak, respectively. This FOM has units of inverse RIUs ( $\left.\mathrm{RIU}^{-1}\right)$. The values obtained for $\mathrm{Cu}$ $\left(\mathrm{Cu} @ \mathrm{Cu}_{2} \mathrm{O}\right) \mathrm{NPs}$ are 2.5 and $6.1 \mathrm{RIU}^{-1}\left(6.3\right.$ and $\left.7.0 \mathrm{RIU}^{-1}\right)$, respectively. Silver nanorods, which are possibly the best available sensors, have a FOM of approximately $100 \mathrm{RIU}^{-1}$, while $\mathrm{Au}$ nanorods have a FOM of $\sim 50 \mathrm{RIU}^{-1}$ [44]. As we can see, the $\mathrm{Cu} @ \mathrm{Cu}_{2} \mathrm{O}$ structures do not compare favorably with them; however, their FOM values are high enough to make these structures viable candidates for use as sensors in some cases where the use of $\mathrm{Ag}$ and $\mathrm{Au}$ is not possible or desired.

Finally, a note of warning is in place here. Cuprous oxide has an excitonic peak located at $\sim 720 \mathrm{~nm}$ [27], which cannot be reproduced by the employed calculation algorithm. This means that for the thicker $\mathrm{Cu}_{2} \mathrm{O}$ shells the plasmonic and excitonic peaks can be degenerate in energy and, in principle, some plasmon-exciton coupling can occur [45]. Though the contribution of these peaks on the optical spectra of $\mathrm{Cu} @ \mathrm{Cu}_{2} \mathrm{O}$ structures cannot be ruled out, it is unlikely to be significant as both peaks are very weak.

\section{CONCLUSIONS}

The linear optical responses of surface-oxidized $\mathrm{Cu}$ NPs have been studied in this work using classical Mie theory, considering their $\mathrm{Cu} @ \mathrm{Cu}_{2} \mathrm{O}$ core-shell type configurations. It has been demonstrated that, contrary to common perception, the surface-oxidized particles might have some advantages for plasmonic applications. First, the position of the SPR can be fine-tuned by varying the thickness of the oxide layer, and, second, it is possible to obtain an enhanced plasmonic response in the oxidized NPs (SPR intensity gains in the order of $25 \%-30 \%$ ) for certain values of oxide layer thickness. Although the electric fields inside these two types of NPs are quite different, they are almost indistinguishable outside, in the suspending medium. Finally, our calculations also suggest that the $\mathrm{Cu} @ \mathrm{Cu}_{2} \mathrm{O}$-type structures are better suited for sensing applications than the pure $\mathrm{Cu}$ NPs.

\section{ACKNOWLEDGMENTS}

O. Peña-Rodríguez thanks the Consejo Nacional de Ciencia y Tecnología for extending a postdoctoral fellowship. The work was partially supported by Vicerrectoría de Investigación y Estudios de Posgrado-Benemérita Universidad Autónoma de Puebla project grant 2011.

\section{REFERENCES}

1. E. Ozbay, "Plasmonics: merging photonics and electronics at nanoscale dimensions," Science 311, 189-193 (2006).

2. S. A. Maier, Plasmonics: Fundamentals and Applications, 1st ed. (Springer, 2007).

3. P. K. Jain, X. Huang, I. H. El-Sayed, and M. A. El-Sayed, "Review of some interesting surface plasmon resonance-enhanced properties of noble metal nanoparticles and their applications to biosystems," Plasmonics 2, 107-118 (2007).

4. H. A. Atwater and A. Polman, "Plasmonics for improved photovoltaic devices,” Nat. Mater. 9, 205-213 (2010).

5. Y. Lei and W.-K. Chim, "Highly ordered arrays of metal/ semiconductor core-shell nanoparticles with tunable nanostructures and photoluminescence,” J. Am. Chem. Soc. 127, 1487-1492 (2005).

6. T. Ghodselahi, M. A. Vesaghi, and A. Shafiekhani, "Study of surface plasmon resonance of $\mathrm{Cu} @ \mathrm{Cu}_{2} \mathrm{O}$ core-shell nanoparticles by Mie theory," J. Phys. D 42, 015308 (2009).

7. L. R. Hirsch, R. J. Stafford, J. A. Bankson, S. R. Sershen, B. Rivera, R. E. Price, J. D. Hazle, N. J. Halas, and J. L. West, "Nanoshell-mediated near-infrared thermal therapy of tumors under magnetic resonance guidance," Proc. Natl. Acad. Sci. USA 100, 13549-13554 (2003).

8. J. Z. Zhang, "Biomedical applications of shape-controlled plasmonic nanostructures: a case study of hollow gold nanospheres for photothermal ablation therapy of cancer," J. Phys. Chem. Lett. 1, 686-695 (2010).

9. Z. Ai, L. Zhang, S. Lee, and W. Ho, "Interfacial hydrothermal synthesis of $\mathrm{Cu} @ \mathrm{Cu}_{2} \mathrm{O}$ core-shell microspheres with enhanced visible-light-driven photocatalytic activity," J. Phys. Chem. C 113, 20896-20902 (2009).

10. S. B. Kalidindi, U. Sanyal, and B. R. Jagirdar, "Nanostructured $\mathrm{Cu}$ and $\mathrm{Cu} @ \mathrm{Cu}_{2} \mathrm{O}$ core shell catalysts for hydrogen generation from ammonia-borane," Phys. Chem. Chem. Phys. 10, 5870-5874 (2008).

11. G. Baffou, R. Quidant, and C. Girard, "Heat generation in plasmonic nanostructures: influence of morphology," Appl. Phys. Lett. 94, 153109 (2009). 
12. S. D. Hudson and G. Chumanov, "Surface enhanced Raman scattering and resonance elastic scattering from capped single Ag nanoparticles,” J. Phys. Chem. C 112, 19866-19871 (2008).

13. L. Gao and X. P. Yu, "Second- and third-harmonic generations for a nondilute suspension of coated particles with radial dielectric anisotropy," Eur. Phys. J. B 55, 403-409 (2007).

14. P. Alivisatos, "The use of nanocrystals in biological detection," Nat. Biotechnol. 22, 47-52 (2004).

15. Z. Jian, Z. Jun-Wu, and L. Jian-Jun, "Location-dependent local field enhancement along the surface of the metal-dielectric core-shell nanostructure," Plasmonics 5, 311-318 (2010).

16. J. Zhu, "Enhanced fluorescence from $\mathrm{Dy}^{3+}$ owing to surface plasmon resonance of $\mathrm{Au}$ colloid nanoparticles," Mater. Lett. 59, 1413-1416 (2005).

17. N. Pinçon, B. Palpant, D. Prot, E. Charron, and S. Debrus, "Third-order nonlinear optical response of $\mathrm{Au}: \mathrm{SiO}_{2}$ thin films: Influence of gold nanoparticle concentration and morphologic parameters," Eur. Phys. J. D 19, 395-402 (2002).

18. Q.-Q. Wang, J.-B. Han, D.-L. Guo, S. Xiao, Y.-B. Han, H.-M. Gong, and X.-W. Zou, "Highly efficient avalanche multiphoton luminescence from coupled $\mathrm{Au}$ nanowires in the visible region," Nano Lett. 7, 723-728 (2007).

19. L. Gao, L. Gu, and Z. Li, "Optical bistability and tristability in nonlinear metal/dielectric composite media of nonspherical particles," Phys. Rev. E 68, 066601 (2003).

20. Y. Liu, Y. Chu, Y. Zhuo, L. Dong, L. Li, and M. Li, "Controlled synthesis of various hollow $\mathrm{Cu}$ nano/microstructures via a novel reduction route," Adv. Funct. Mater. 17, 933-938 (2007).

21. J. R. Hayes, G. W. Nyce, J. D. Kuntz, J. H. Satcher, and A. V. Hamza, "Synthesis of bi-modal nanoporous $\mathrm{Cu}, \mathrm{CuO}$ and $\mathrm{Cu}_{2} \mathrm{O}$ monoliths with tailored porosity," Nanotechnology 18, 275602 (2007).

22. C. Sönnichsen and A. P. Alivisatos, "Gold nanorods as novel nonbleaching plasmon-based orientation sensors for polarized single-particle microscopy," Nano Lett. 5, 301-304 (2005).

23. G. Raschke, S. Brogl, A. S. Susha, A. L. Rogach, T. A. Klar, J. Feldmann, B. Fieres, N. Petkov, T. Bein, A. Nichtl, and K. Kurzinger, "Gold nanoshells improve single nanoparticle molecular sensors," Nano Lett. 4, 1853-1857 (2004).

24. A. D. McFarland and R. P. Van Duyne, "Single silver nanoparticles as real-time optical sensors with zeptomole sensitivity," Nano Lett. 3, 1057-1062 (2003).

25. H. Xu and M. Käll, "Surface-plasmon-enhanced optical forces in Silver nanoaggregates,” Phys. Rev. Lett. 89, 246802 (2002).

26. M. Yin, C.-K. Wu, Y. Lou, C. Burda, J. T. Koberstein, Y. Zhu, and S. O’Brien, “Copper oxide nanocrystals,” J. Am. Chem. Soc. 127, 9506-9511 (2005).

27. K. P. Rice, E. J. Walker, M. P. Stoykovich, and A. E. Saunders, "Solvent-dependent surface plasmon response and oxidation of copper nanocrystals," J. Phys. Chem. C 115, 1793-1799 (2011).

28. S. J. Pearton, C. R. Abernathy, M. E. Overberg, G. T. Thaler, D. P. Norton, N. Theodoropoulou, A. F. Hebard, Y. D. Park, F. Ren,
J. Kim, and L. A. Boatner, "Wide band gap ferromagnetic semiconductors and oxides," J. Appl. Phys. 93, 1-13 (2003).

29. G. H. Chan, J. Zhao, E. M. Hicks, G. C. Schatz, and R. P. Van Duyne, "Plasmonic properties of copper nanoparticles fabricated by nanosphere lithography," Nano Lett. 7, 1947-1952 (2007).

30. L.-Y. Shao, J. P. Coyle, S. T. Barry, and J. Albert, "Anomalous permittivity and plasmon resonances of copper nanoparticle conformal coatings on optical fibers," Opt. Mater. Express 1, 128-137 (2011).

31. D.-K. Kim, S. M. Yoo, T. J. Park, H. Yoshikawa, E. Tamiya, J. Y. Park, and S. Y. Lee, "Plasmonic properties of the multispot copper-capped nanoparticle array chip and its application to optical biosensors for pathogen detection of multiplex DNAs," Anal. Chem. 83, 6215-6222 (2011).

32. G. Mie, "Beiträge zur Optik trüber medien, speziell kolloidaler Metallösungen," Ann. Phys. 330, 377-445 (1908).

33. C. F. Bohren and D. R. Huffman, Absorption and Scattering of Light by Small Particles (Wiley-Interscience, 1998).

34. O. Peña and U. Pal, "Scattering of electromagnetic radiation by a multilayered sphere," Comput. Phys. Commun. 180, 2348-2354 (2009).

35. P. B. Johnson and R. W. Christy, "Optical constants of the noble metals," Phys. Rev. B 6, 4370-4379 (1972).

36. R. D. Averitt, S. L. Westcott, and N. J. Halas, "Linear optical properties of gold nanoshells,” J. Opt. Soc. Am. B 16, 1824-1832 (1999).

37. O. Peña, U. Pal, L. Rodríguez-Fernández, and A. Crespo-Sosa, "Linear optical response of metallic nanoshells in different dielectric media," J. Opt. Soc. Am. B 25, 1371-1379 (2008).

38. E. D. Palik, Handbook of Optical Constants of Solids (Academic, 1997).

39. W. Yang, "Improved recursive algorithm for light scattering by a multilayered sphere," Appl. Opt. 42, 1710-1720 (2003).

40. A. E. Neeves and M. H. Birnboim, "Composite structures for the enhancement of nonlinear-optical susceptibility," J. Opt. Soc. Am. B 6, 787-796 (1989).

41. H. Wang, F. Tam, N. K. Grady, and N. J. Halas, "Cu nanoshells effects of interband transitions on the nanoparticle plasmon resonance," J. Phys. Chem. B 109, 18218-18222 (2005).

42. Z. Liu and Y. Bando, "A novel method for preparing copper nanorods and nanowires," Adv. Mater. 15, 303-305 (2003).

43. L. J. Sherry, S.-H. Chang, G. C. Schatz, R. P. Van Duyne, B. J. Wiley, and Y. Xia, "Localized surface plasmon resonance spectroscopy of single silver nanocubes," Nano Lett. 5, 2034-2038 (2005).

44. O. Peña-Rodríguez, U. Pal, V. Rodríguez-Iglesias, L. RodríguezFernández, and A. Oliver, "Configuring Au and Ag nanorods for sensing applications,” J. Opt. Soc. Am. B 28, 714-720 (2011).

45. N. T. Fofang, T.-H. Park, O. Neumann, N. A. Mirin, P. Nordlander, and N. J. Halas, "Plexcitonic nanoparticles: plasmon-exciton coupling in nanoshell-J-aggregate complexes," Nano Lett. 8, 3481-3487 (2008). 